\title{
Criticality of Measures on 2-d Ising Configurations: From Square to Hexagonal Graphs
}

\author{
Valentina Apollonio ${ }^{1} \cdot$ Roberto $^{\prime}$ Autilia $^{1} \cdot$ Benedetto Scoppola $^{2}$ (D) \\ Elisabetta Scoppola ${ }^{1}$ - Alessio Troiani ${ }^{3}$
}

Received: 6 June 2019 / Accepted: 9 October 2019 / Published online: 23 October 2019

(c) Springer Science+Business Media, LLC, part of Springer Nature 2019

\begin{abstract}
On the space of Ising configurations on the 2-d square lattice, we consider a family of non Gibbsian measures introduced by using a pair Hamiltonian, depending on an additional inertial parameter $q$. These measures are related to the usual Gibbs measure on $\mathbb{Z}^{2}$ and turn out to be the marginal of the Gibbs measure of a suitable Ising model on the hexagonal lattice. The inertial parameter $q$ tunes the geometry of the system. The critical behaviour and the decay of correlation functions of these measures are studied thanks to relation with the Random Cluster model. This measure turns out to be interesting also because it is the stationary measure of a class of Probabilistic Cellular Automata (PCA). Such PCA can be used to obtain a fast sample of the Ising measures on 2-d lattices.
\end{abstract}

Keywords Ising model $\cdot$ Random cluster model $\cdot$ Phase transitions $\cdot$ Correlation functions

\section{Introduction and Definitions}

Let $\Lambda$ be a two-dimensional $2 L \times 2 L$ square box in $\mathbb{Z}^{2}$ centered at the origin and let $\mathcal{B}_{\Lambda}$ denote the set of all nearest neighbours in $\Lambda$ assuming periodic boundary conditions. In other words $\mathcal{B}_{\Lambda}$ consists of all pairs $\{\langle x, y\rangle: x, y \in \Lambda,|x-y|=1\}$, with $|x-y|$ being the usual lattice distance in $\mathbb{Z}^{2}$, plus the pairs of sites at opposite faces of the square $\Lambda$. We denote by $\mathcal{X}_{\Lambda}$ the set of spin configurations in $\Lambda$, i.e., $\mathcal{X}_{\Lambda}=\{-1,1\}^{\Lambda}$. On this spin configuration space we consider the nearest neighbours ferromagnetic Ising Hamiltonian

Communicated by Eric A. Carlen.

$\triangle$ Benedetto Scoppola

scoppola@mat.uniroma2.it

1 Dipartimento di Matematica e Fisica, Università Roma Tre, Largo San Murialdo, 1, 00146 Rome, Italy

2 Dipartimento di Matematica, Università di Roma "Tor Vergata", Via della Ricerca Scientifica, 1, 00133 Rome, Italy

3 Dipartimento di Matematica “Tullio Levi-Civita”, Università di Padova, Via Trieste, 63, 35121 Padua, Italy 


$$
H(\sigma)=-\sum_{\langle x, y\rangle \in \mathcal{B}_{\Lambda}} J \sigma_{x} \sigma_{y}
$$

with $J>0$ and the associated Gibbs measure

$$
\pi^{G}(\sigma):=\frac{1}{Z^{G}} e^{-H(\sigma)} \quad \text { with } \quad Z^{G}=\sum_{\sigma \in \mathcal{X}_{\Lambda}} e^{-H(\sigma)} .
$$

Looking for efficient algorithms to sample from this measure, an approximate sampling by means of a pair Hamiltonian, adaptable to general pair interaction, has been introduced in [5]. The main idea was, indeed, to define a parallel dynamics, i.e., a Markov chain updating all spins at each time, with an invariant measure strictly related to $\pi^{G}$. Following these ideas, a non reversible parallel dynamics with polynomial mixing time in the size of the system has been the subject of a successive paper [6] where the main ingredient was the combination of parallel updating and non symmetric interaction.

Define the space of pairs of configurations

$$
\mathcal{X}_{\Lambda}^{2}=\mathcal{X}_{\Lambda} \times \mathcal{X}_{\Lambda}
$$

For each pair $(\sigma, \tau) \in \mathcal{X}_{\Lambda}^{2}$ we define the Hamiltonian with asymmetric interaction

$$
H(\sigma, \tau)=-\sum_{x \in \Lambda}\left[J \sigma_{x}\left(\tau_{x \uparrow}+\tau_{x \rightarrow}\right)+q \sigma_{x} \tau_{x}\right]=-\sum_{x \in \Lambda}\left[J \tau_{x}\left(\sigma_{x \downarrow}+\sigma_{x} \leftarrow\right)+q \tau_{x} \sigma_{x}\right]
$$

where $x^{\uparrow}, x^{\rightarrow}, x^{\downarrow}, x^{\leftarrow}$ are respectively the up, right, down, left neighbours of the site $x$ on the torus $\left(\Lambda, \mathcal{B}_{\Lambda}\right), J>0$ is the ferromagnetic interaction and $q>0$ is an inertial constant. It is straightforward to see that $H(\sigma, \sigma)=H(\sigma)-q|\Lambda|$ where $H(\sigma)$ is the Ising Hamiltonian given in (1). Note also that $H(\sigma, \tau) \neq H(\tau, \sigma)$.

On the configuration space $\mathcal{X}_{\Lambda}$ we define the following family of measures, indexed by $q$ :

$$
\pi_{q}(\sigma)=\frac{1}{Z} \sum_{\tau \in \mathcal{X}_{\Lambda}} e^{-H(\sigma, \tau)} \quad \text { with } \quad Z=\sum_{(\sigma, \tau) \in \mathcal{X}_{\Lambda}^{2}} e^{-H(\sigma, \tau)} .
$$

These measures have been considered in the previous papers $[5,6,10]$ and turn out to be the invariant measure of the parallel dynamics defined there. In a more recent paper [3] $\pi_{q}(\sigma)$ is the invariant measure of a reversible parallel dynamics, the "shaken dynamics", that can be used to model geological processes related to earthquakes for a suitable choice of the parameter $q$.

The goal of the present paper is to study, from a static point of view, the thermodynamical properties and the critical behaviour of this family of probability measures. This analysis is performed relating $\pi_{q}$ to the Gibbs measure of the Ising model on a different lattice induced by the pair Hamiltonian and using the standard coupling between Ising model and Random Cluster Model (RCM). In this context we will show that the parameter $q$ tunes the geometry of the lattice.

In the remainder of the paper, in order to lighten the notation, we write $\pi$ in place of $\pi_{q}$.

The usual Gibbs measure (2) and the measure $\pi(\sigma)$ defined above are connected by the following result obtained in $[5,10]$ (see Theorem 1.2 in [10]):

Theorem 1.1 Define the total variation distance, or $L_{1}$ distance, between $\pi$ and $\pi^{G}$ as

$$
\left\|\pi-\pi^{G}\right\|_{T V}=\frac{1}{2} \sum_{\sigma \in \mathcal{X}_{\Lambda}}\left|\pi(\sigma)-\pi^{G}(\sigma)\right| .
$$


Fig. 1 Interaction in the pair Hamiltonian

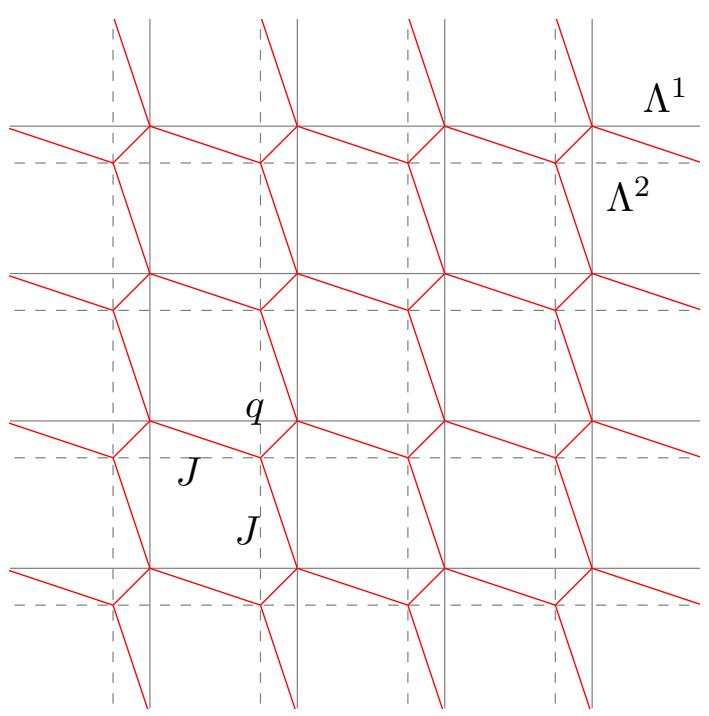

Set $\delta=e^{-2 q}$, and let $\delta$ be such that

$$
\lim _{|\Lambda| \rightarrow \infty} \delta^{2}|\Lambda|=0
$$

then there exists $\bar{J}$ such that for any $J>\bar{J}$

$$
\lim _{|\Lambda| \rightarrow \infty}\left\|\pi-\pi^{G}\right\|_{T V}=0
$$

Let us observe that the pair Hamiltonian (3), considering only half of the interactions (down-left), allows to interpolate between different lattices. Indeed, as already shown in [3], the space of pairs of configurations with interaction given by $H(\sigma, \tau)$ can be represented as the configuration space $\mathcal{X}_{\mathbb{H}}$ for the Ising model on an hexagonal lattice $\mathbb{H}=(V, E)$. Indeed, the hexagonal lattice $\mathbb{H}$ is obtained by considering two copies $\Lambda^{1}$ and $\Lambda^{2}$ of $\Lambda$ and associating each vertex $x \in \Lambda$ to the pair $\left(x^{1} \in \Lambda^{1}, x^{2} \in \Lambda^{2}\right)$. Setting $\sigma=\left(\sigma^{1}, \sigma^{2}\right)$ with $\sigma^{i} \in \mathcal{X}_{\Lambda^{i}}, i=$ 1,2 , and considering the interaction defined by $H\left(\sigma^{1}, \sigma^{2}\right)$ it is straightforward to observe that $\mathbb{H}$ is a bipartite graph. On this graph we distinguish two types of edges and set $E=E_{J} \cup E_{q}$. Indeed two of the three edges exiting from each site correspond to the left and downwards interactions of strength $J$ (in the set $E_{J}$ ), while the third corresponds to the self-interaction $q$ (in the set $E_{q}$ ) (Fig. 1).

In other words we associate to each edge $e$ a weight

$$
\mathrm{J}_{e}= \begin{cases}J & \text { if } e \in E_{J} \\ q & \text { if } e \in E_{q}\end{cases}
$$

We define the Gibbs measure for configurations $\sigma=\left(\sigma^{1}, \sigma^{2}\right)$ on the hexagonal lattice

$$
\pi_{2}\left(\sigma^{1}, \sigma^{2}\right)=\frac{e^{-H\left(\sigma^{1}, \sigma^{2}\right)}}{Z}
$$


where the pair Hamiltonian, defined in (3), has been written as

$$
H\left(\sigma^{1}, \sigma^{2}\right)=-\sum_{e \in E} \mathrm{~J}_{e} \sigma_{e^{1}}^{1} \sigma_{e^{2}}^{2}
$$

with $e^{1}, e^{2}$ the two sites in $\mathbb{H}$ connected by the edge $e$. Exploiting this representation, we can apply to our model the powerful connection between Ising model and Random Cluster Model.

Assume periodic boundary conditions and define $\Omega:=\{0,1\}^{E}$. For any $\omega \in \Omega$ the edge $e$ is open (or present) if $\omega(e)=1$. Let $\eta(\omega):=\{e \in E: \omega(e)=1\}$ and let $k(\omega):=k(\eta(\omega))$ denote the number of connected components (or open clusters) of the graph $(V, \eta(\omega))$. Given now two parameters $p_{J}, p_{q} \in[0,1]$, by defining

$$
p_{e}= \begin{cases}p_{J} & \text { if } e \in E_{J} \\ p_{q} & \text { if } e \in E_{q}\end{cases}
$$

we introduce the measure on $\Omega$ :

$$
\Phi_{p_{e}}(\omega)=\frac{1}{Z^{R C}}\left\{\prod_{e \in E} p_{e}^{\omega(e)}\left(1-p_{e}\right)^{1-\omega(e)}\right\} 2^{k(\omega)}
$$

with partition function

$$
Z^{R C}=\sum_{\omega \in \Omega}\left\{\prod_{e \in E} p_{e}^{\omega(e)}\left(1-p_{e}\right)^{1-\omega(e)}\right\} 2^{k(\omega)} .
$$

Following the general theory (see for instance [9]) we define now a coupling between our pairs of configurations $\sigma=\left(\sigma^{1}, \sigma^{2}\right) \in \mathcal{X}_{\Lambda}^{2}$ and the random cluster configuration $\omega \in \Omega$ by the following probability mass on $\mathcal{X}_{\Lambda}^{2} \times \Omega$ :

$$
\mu(\boldsymbol{\sigma}, \omega) \propto \prod_{e \in E}\left\{\left(1-p_{e}\right) \delta_{\omega(e), 0}+p_{e} \delta_{\omega(e), 1} \delta_{e}(\boldsymbol{\sigma})\right\}
$$

where

$$
\delta_{e}(\sigma)=\delta_{\sigma_{x}^{1}, \sigma_{y}^{2}} \text { for } \quad e=(x, y), \text { with } x \in \Lambda^{1}, y \in \Lambda^{2}
$$

We have the following result:

Proposition 1.2 If $p_{J}=1-e^{-2 J}$ and $p_{q}=1-e^{-2 q}$

(1) The marginal on $\mathcal{X}_{\Lambda}^{2}$ of $\mu(\sigma, \omega)$ is

$$
\mu_{1}(\boldsymbol{\sigma})=\sum_{\omega \in \Omega} \mu((\sigma), \omega)=\pi_{2}\left(\sigma^{1}, \sigma^{2}\right)
$$

(2) The marginal on $\Omega$ of $\mu(\sigma, \omega)$ is

$$
\mu_{2}(\omega)=\sum_{\sigma} \in \mathcal{X}_{\Lambda, B}^{2} \mu(\sigma, \omega)=\Phi_{p_{e}}(\omega)
$$

(3) The conditional measure on $\mathcal{X}_{\Lambda}^{2}$ given $\omega$ is obtained by putting uniformly random spins on entire clusters of $\omega$. These spins are constant on given clusters, are independent between clusters and each is uniformly distributed on the set $\{-1,+1\}$.

(4) The conditional measure on $\Omega$ given $\sigma$ is obtained by setting $\omega(e)=0$ if $\delta_{e}(\sigma)=0$ and otherwise $\omega(e)=1$ with probability $p_{J}\left(p_{q}\right)$ for $e \in E_{J}\left(e \in E_{q}\right)$. 
For the proof of this proposition we refer to the clear review by Grimmett [9] of the FortuinKasteleyn construction [8], and to the rich papers [1] and [7] for further developments. The coupling between these two models is robust and of wide applicability, in particular in [9] the infinite-volume random-cluster measure and phase transitions are widely discussed. With this construction we can easily prove that our model exhibits a phase transition and we can compute the strong anisotropy of the correlation functions.

Our results are presented in the next section and are proven in Sect. 3. In the final section we describe some numerical aspects. In what follows, for any $x, y \in V$ we will denote by $\{x \leftrightarrow y\}$ the set of $\omega \in \Omega$ for which there exists an open path joining the vertex $x$ with the vertex $y$.

\section{Results}

The measure $\pi$, although not Gibbsian, turns out to be the marginal of the Gibbs measure $\pi_{2}$ of the Ising model of the hexagonal lattice and inherits from it the thermodynamics. In other words we can extend to the non Gibbsian measure $\pi$ thermodynamical relations and the control of the critical behaviour obtained for the measure $\pi_{2}$. To obtain these results we leverage on the well established random cluster coupling.

The first result relates the thermodynamical properties of the measures $\pi$ and $\pi_{2}$.

Theorem 2.1 Consider the measure $\pi$ defined in (4) as the marginal of the Gibbs measure on the hexagonal lattice

$$
\pi_{2}\left(\sigma^{1}, \sigma^{2}\right)=\frac{1}{Z} e^{-H\left(\sigma^{1}, \sigma^{2}\right)}
$$

with the same partition function

$$
Z=\sum_{\left(\sigma^{1}, \sigma^{2}\right) \in \mathcal{X}_{\Lambda}^{2}} e^{-H\left(\sigma^{1}, \sigma^{2}\right)} .
$$

The following relations hold:

(1) The average magnetization with respect to the measure $\pi$ and $\pi_{2}$ is the same, that is

$$
m:=\pi\left(\frac{\sum_{x \in \Lambda} \sigma_{x}}{|\Lambda|}\right)=m_{2}:=\pi_{2}\left(\frac{\sum_{x \in \Lambda^{1} \cup \Lambda^{2}} \sigma_{x}}{2|\Lambda|}\right)
$$

(2) Let $\pi^{+}\left(\pi^{-}\right)$and $\pi_{2}^{+}\left(\pi_{2}^{-}\right)$be the previous measures with plus (minus) boundary conditions, then for any $x \in \Lambda$

$$
\pi^{ \pm}\left(\sigma_{x}\right)= \pm \Phi_{p_{e}}\left(x^{1} \leftrightarrow \partial \Lambda^{1}\right)
$$

(3) For any $x, y \in \Lambda$

$$
\pi\left(\sigma_{x} \sigma_{y}\right)=\Phi_{p_{e}}\left(x^{1} \leftrightarrow y^{1}\right)
$$

with the obvious notation $x^{1}, y^{1} \in \Lambda^{1}$ for the sites in the layer $\Lambda^{1}$ corresponding to vertices $x$ and $y$ in $\Lambda$, respectively.

In our second theorem we identify the critical behaviour of the system.

Theorem 2.2 The critical equation relating the parameters $J$ and $q$ in the measure $\pi$ is given by the equation: 
Fig. 2 The function $J_{c}(q)$

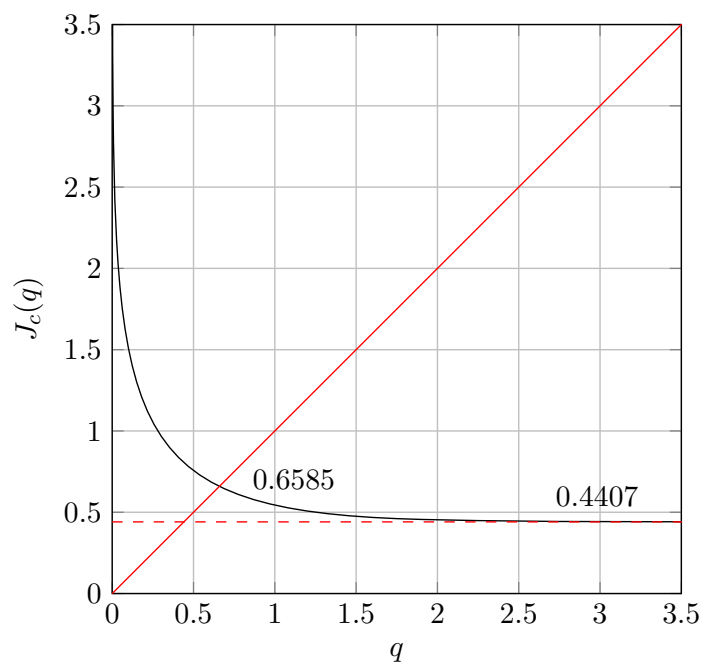

$$
J_{c}(q)=\tanh ^{-1}\left(-\tanh q+\sqrt{\tanh ^{2} q+1}\right)
$$

Remark 2.3 It is well known that the Gibbs measure $\pi^{G}$ on the square lattice exhibits a phase transition at

$$
J_{c}^{G}=\tanh ^{-1}(\sqrt{2}-1)=0.4407 \ldots
$$

Note that

$$
\lim _{q \rightarrow \infty} J_{c}(q)=J_{c}^{G}
$$

Furthermore, the curve $J_{c}(q)$ intersects the line $J=q$ for $J=\tanh ^{-1}\left(\frac{\sqrt{3}}{3}\right)=0.6585 \ldots$, corresponding to the critical value of $J$ in the homogeneous hexagonal lattice (see Fig. 2).

The parameter $q$ tunes the geometry of the system. In fact the limit $q \rightarrow 0$ corresponds to erasing the $q$-edges obtaining, from the hexagonal lattice, independent copies of 1- $d$ Ising model. Indeed for $q \rightarrow 0$ we find $J_{c} \rightarrow \infty$ showing the absence of phase transition for the one-dimensional Ising model. The opposite limit, $q \rightarrow \infty$, corresponds to the collapse of the hexagonal lattice into the square one, by identifying the sites connected by the $q$-edges. The case $J=q$ corresponds to the homogeneuous hexagonal graph.

The next and last result is about correlation functions and reflects the anisotropy of the model, depending on the parameter $q$.

Theorem 2.4 If the parameter $q$ is sufficiently small, for any integer $\ell \in(0, L)$ there exist two constants $c_{1}<c_{2}$ such that

$$
\pi\left(\sigma_{(0,0)} \sigma_{(\ell, \ell)}\right) \leq c_{1}<c_{2} \leq \pi\left(\sigma_{(0, \ell)} \sigma_{(\ell, 0)}\right) .
$$




\section{Proof of the Results}

\subsection{Proof of Theorem 2.1}

(1) The statement immediately follows from direct computation, indeed:

$$
m=\sum_{\sigma} \frac{\sum_{x \in \Lambda} \sigma_{x}}{|\Lambda|} \cdot \sum_{\tau} \frac{e^{-H(\sigma, \tau)}}{Z}=\frac{1}{2} \sum_{(\sigma, \tau)} \frac{\sum_{x \in \Lambda}\left(\sigma_{x}+\tau_{x}\right)}{|\Lambda|} \cdot \frac{e^{-H(\sigma, \tau)}}{Z}=m_{2}
$$

where the second equality follows by a symmetry argument.

(2) The standard coupling between Ising and the RCM on $\mathbb{H}$ yields

$$
\begin{aligned}
\pi^{+}\left(\sigma_{x}\right) & =\sum_{\sigma} \sigma_{x} \pi^{+}(\sigma)=\sum_{\sigma} \sigma_{x^{1}}^{1} \pi_{2}^{+}(\sigma)=\pi_{2}^{+}\left(\sigma_{x^{1}}^{1}\right) \\
& =\sum_{\omega \in \Omega} \sum_{\sigma} \mu(\sigma, \omega) \sigma_{x^{1}}^{1}\left(\mathbb{1}_{x^{1} \leftrightarrow \partial \Lambda^{1}}+\mathbb{1}_{x^{1} \leftrightarrow \partial \Lambda^{1}}\right) \\
& =\Phi_{p_{e}}\left(x^{1} \leftrightarrow \partial \Lambda^{1}\right)+\sum_{\omega \in \Omega} \sum_{\sigma}\left[\mu(\sigma, \omega \mid \omega) \sigma_{x^{1}}^{1} \mathbb{1}_{x^{1} \leftrightarrow \partial \Lambda^{1}}\right] \Phi_{p_{e}}(\omega) \\
& =\Phi_{p_{e}}\left(x^{1} \leftrightarrow \partial \Lambda^{1}\right)
\end{aligned}
$$

since by proposition 1.2 the square bracket vanishes. The minus boundary conditions can be treated in the same way.

(3) The proof of point (3) can be obtained following the same argument.

\subsection{Proof of Theorem 2.2}

As shown in [4], for a planar weighted graph $G=(V, E)$ that is non degenerate, finite and doubly periodic, the critical curve of the Hamiltonian

$$
H(\sigma)=-\sum_{e=\{u, v\} \in E} \mathrm{~J}_{e} \sigma_{u} \sigma_{v}
$$

is the unique solution of the equation

$$
\sum_{\gamma \in \mathcal{E}_{0}(G)} \prod_{e \in \gamma} \tanh \mathrm{J}_{e}=\sum_{\gamma \in \mathcal{E}_{1}(G)} \prod_{e \in \gamma} \tanh \mathrm{J}_{e}
$$

where $\mathcal{E}(G)$ is the set of the even subgraphs of $G$, i.e., the set of subgraphs $\gamma$ of $G$ such that each vertex of $G$ is an endvertex of an even number of edges of $\gamma, \mathcal{E}_{0}(G)$ is the set of the even subgraphs of $G$ winding an even number of times around each of the two dimensions of the torus and $\mathcal{E}_{1}(G)=\mathcal{E}(G) \backslash \mathcal{E}_{0}(G)$. The main step in the proof of this result is to show that the free energy per fundamental domain can be expressed in terms of the Kac-Ward determinants.

In our case the hexagonal lattice $\mathbb{H}=(V, E)$ satisfies the conditions of the theorem 1.1 in [4] and the equation (14) can be obtained by periodically glueing on the torus the cell represented in Fig. 3.

The explicit form of the equation is

$$
1=2 \tanh J \tanh q+\tanh ^{2} J
$$



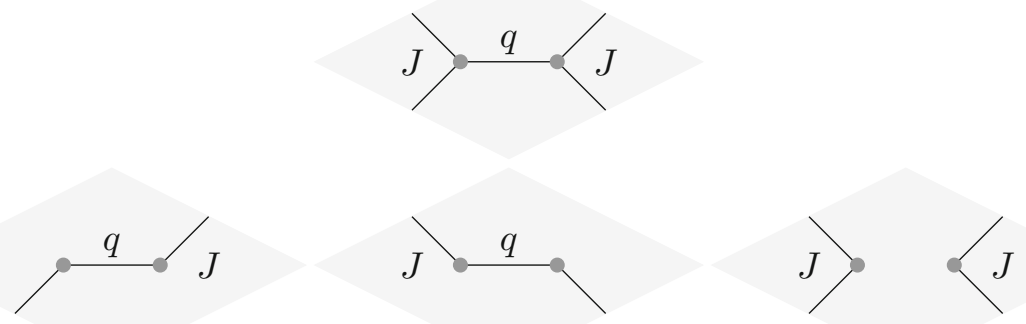

Fig. 3 The elementary cell on the torus and the three corresponding even subgraphs $\gamma \in \mathcal{E}_{1}$

where on the r.h.s. we have the sum of the contributions from the three even subgraphs in $\mathcal{E}_{1}$ shown in Fig. 3 while the 1 on the 1.h.s. is the contribution of the unique graph in $\mathcal{E}_{0}$ without edges.

Solving Eq. (15) w.r.t. $J$ gives the curve

$$
J_{c}(q)=\tanh ^{-1}\left(\sqrt{\tanh ^{2} q+1}-\tanh q\right)
$$

represented in Fig. 2

\subsection{Proof of Theorem 2.4}

Let $\gamma \subset E$ be a path of open edges connecting two vertices $x^{1}, y^{1} \in \Lambda^{1}$. We introduce the notation $\eta(\omega) \supset \gamma$ to identify all the configurations $\omega \in \Omega$ such that $\omega(e)=1, \forall e \in \gamma$. By definition

$$
\begin{aligned}
\Phi_{p_{e}}\left(x^{1} \leftrightarrow y^{1}\right) & =\sum_{\gamma: x^{1} \leftrightarrow y^{1}} \sum_{\substack{\omega \in \Omega: \\
\eta(\omega) \supset \gamma}} \Phi_{p_{e}}(\omega) \\
& =\frac{1}{Z^{R C}} \sum_{\gamma: x^{1} \leftrightarrow y^{1}}\left(\prod_{e \in \gamma} p_{e}\right) \sum_{\omega^{\prime} \in\{0,1\}^{E \backslash \gamma}}\left(\prod_{e \in E \backslash \gamma} p_{e}^{\omega^{\prime}(e)}\left(1-p_{e}\right)^{1-\omega^{\prime}(e)}\right) 2^{k\left(\eta\left(\omega^{\prime}\right) \cup \gamma\right)} \\
& =\frac{1}{Z^{R C}} \sum_{\gamma: x^{1} \leftrightarrow y^{1}}\left(\prod_{e \in \gamma} p_{e}\right) Z_{\gamma}
\end{aligned}
$$

where

$$
Z_{\gamma}=\sum_{\omega^{\prime} \in\{0,1\}^{E \backslash \gamma}}\left(\prod_{e \in E \backslash \gamma} p_{e}^{\omega^{\prime}(e)}\left(1-p_{e}\right)^{1-\omega^{\prime}(e)}\right) 2^{k\left(\eta\left(\omega^{\prime}\right) \cup \gamma\right)}
$$

\section{Upper Bound}

For any path $\gamma$ and any configuration $\omega \in \Omega$ we denote by $\omega^{\prime}$ the restriction of $\omega$ to the set of edges in $E \backslash \gamma$ and by $\omega^{\prime \prime}$ its restriction to the set of edges in $\gamma$.

Since $k(\eta(\omega)) \geq k\left(\eta\left(\omega^{\prime}\right) \cup \gamma\right)$ we can state the following inequality for the partition function 


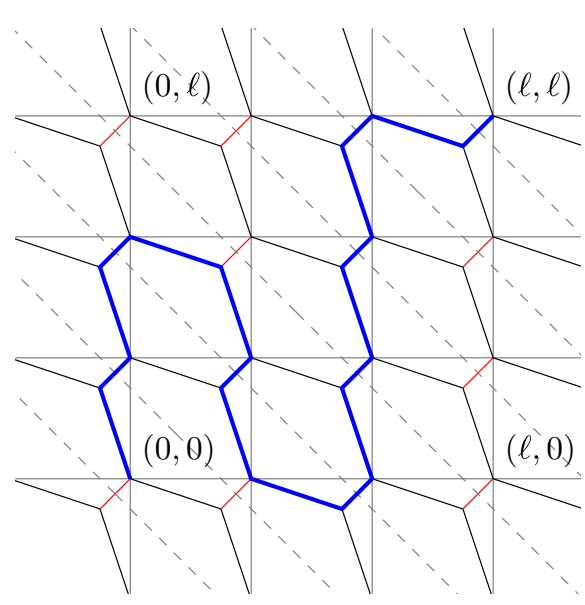

(a)

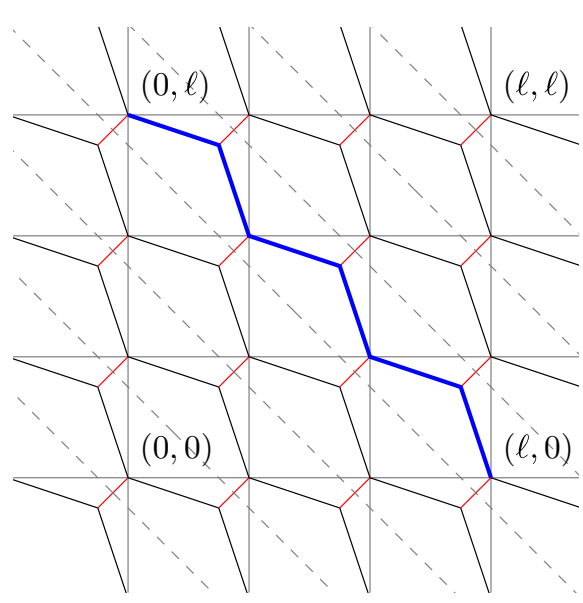

(b)

Fig. 4 Lattice $\mathbb{H}$ with the slices used for the estimation of the correlation functions. Picture a shows an example of path $\gamma:(0,0) \leftrightarrow(\ell, \ell)$. Picture $\mathbf{b}$ shows the diagonal path $\gamma^{\star}$

$$
\begin{aligned}
Z^{R C} & \geq \sum_{\omega \in \Omega}\left(\prod_{e \in \gamma} p_{e}^{\omega(e)}\left(1-p_{e}\right)^{1-\omega(e)}\right)\left(\prod_{e \in E \backslash \gamma} p_{e}^{\omega(e)}\left(1-p_{e}\right)^{1-\omega(e)}\right) 2^{k\left(\eta\left(\omega^{\prime}\right) \cup \gamma\right)} \\
& =\left(\sum_{\omega^{\prime \prime} \in\{0,1\}|\gamma|} \prod_{e \in \gamma} p_{e}^{\omega^{\prime \prime}(e)}\left(1-p_{e}\right)^{1-\omega^{\prime \prime}(e)}\right) Z_{\gamma}=Z_{\gamma}
\end{aligned}
$$

This observation implies

$$
\Phi_{p_{e}}\left(x^{1} \leftrightarrow y^{1}\right) \leq \sum_{\gamma: x^{1} \leftrightarrow y^{1}}\left(\prod_{e \in \gamma} p_{e}\right)
$$

Now let us suppose to slice the lattice $\mathbb{H}$ as in Fig. 4. It is easy to see that each path $\gamma:(0,0) \leftrightarrow(\ell, \ell)$ must visit all slices separating $(0,0)$ and $(\ell, \ell)$ and therefore it crosses at least $2 \ell q$-edges (see Fig. 4(a)). We give an upper bound for the sum in (17) in terms of possible crossing-paths that start in $(0,0)$ and stop in the slice which contains $(\ell, \ell)$. The transition from one slice to the other is determined by the crossing of a $q$-edge. After a $q$-edge has been crossed the path must traverse an arbitrary number of $J$-edges, either on the left or on the right, before crossing the next $q$-edge. Denoting by $\Gamma(n, 2 \ell)$ the number of one dimensional random walks between slices of length $n$ arriving at distance $2 \ell$ from the origin, we can write

$$
\begin{aligned}
\Phi_{p_{e}}((0,0) \leftrightarrow(\ell, \ell)) & \leq \sum_{n=2 \ell}^{\infty} \Gamma(n, 2 \ell)\left(2 p_{q} p_{J}\right)^{n}\left(\sum_{m=0}^{\infty} p_{J}^{m}\right)^{n}=\sum_{n=2 \ell}^{\infty} \Gamma(n, 2 \ell)\left(2 p_{q} p_{J}\right)^{n}\left(\frac{1}{1-p_{J}}\right)^{n} \\
& =\sum_{n=2 \ell}^{\infty}\left(\begin{array}{c}
n \\
\frac{n+2 \ell}{2}
\end{array}\right)\left(\frac{2 p_{q} p_{J}}{1-p_{J}}\right)^{n} \leq \sum_{n=2 \ell}^{\infty}\left(\frac{4 p_{q} p_{J}}{1-p_{J}}\right)^{n} .
\end{aligned}
$$

The last sum converges if $q$ is sufficiently small so that the parameters $p_{J}$ and $p_{q}$ satisfy the condition $\frac{4 p_{q} p_{J}}{1-p_{J}}<1$ and we get 


$$
c_{1}=\frac{\left(\frac{4 p_{q} p_{J}}{1-p_{J}}\right)^{2 \ell}}{1-\left(\frac{4 p_{q} p_{J}}{1-p_{J}}\right)} .
$$

\section{Lower Bound}

We introduce the diagonal path $\gamma^{*}$ connecting $(0, \ell)$ and $(\ell, 0)$ remaining in the same slice as in Fig. 4(b) and $\bar{\gamma}=\gamma^{*} \cup \partial \gamma^{*}$. Let $Z_{E \backslash \bar{\gamma}}$ be the partition function of the Random Cluster Model defined on the graph $\mathbb{H}_{\bar{\gamma}}=(V, E \backslash \bar{\gamma})$. By Theorem (3.60) in [9] we have that $Z_{E \backslash \bar{\gamma}} \geq Z_{E}=Z^{R C}$ and hence we can give a lower bound for the correlation function as follows

$$
\begin{aligned}
& \Phi_{p_{e}}((0, \ell) \leftrightarrow(\ell, 0)) \geq \\
& \geq \frac{1}{Z^{R C}}\left(\prod_{e \in \gamma^{*}} p_{e}\right) \sum_{\omega^{\prime \prime} \in\{0,1\} E \backslash \bar{\gamma}}\left[\prod_{e \in \partial \gamma^{*}}\left(1-p_{e}\right)\right]\left[\prod_{e \in E \backslash \bar{\gamma}} p_{e}^{\omega^{\prime \prime}(e)}\left(1-p_{e}\right)^{1-\omega^{\prime \prime}(e)}\right] 2^{k\left(\omega^{\prime \prime}\right)+1} \\
& =\frac{1}{Z^{R C}}\left(\prod_{e \in \gamma^{*}} p_{e}\right)\left[\prod_{e \in \partial \gamma^{*}}\left(1-p_{e}\right)\right] 2 Z_{E \backslash \bar{\gamma}} \geq 2\left(\prod_{e \in \gamma^{*}} p_{e}\right)\left[\prod_{e \in \partial \gamma^{*}}\left(1-p_{e}\right)\right] \\
& =2 e^{-4 J}\left(1-e^{-2 J}\right)^{2 \ell} e^{-2 q(2 \ell+1)}=c_{2} .
\end{aligned}
$$

If $q$ is sufficiently small such that, for instance,

$$
\frac{4 p_{q}}{\left(1-p_{q}\right)\left(1-p_{J}\right)}<\frac{1}{2}
$$

and

$$
\left(\frac{p_{q}}{1-p_{q}}\right)^{2}<\frac{\left(1-p_{J}\right)^{3}\left(1-p_{q}\right)}{16},
$$

we immediately get $c_{1}<c_{2}$. Note that the first of these two conditions is stronger than $\frac{4 p_{q} p_{J}}{1-p_{J}}<1$ and, therefore, $c_{1}$ is well defined.

\section{Numerical Indications}

The measure $\pi$ is not a Gibbs measure. However it is possible to sample from it in an effective way by drawing samples from the Gibbs measure $\pi_{2}$. Remarkably, this sampling can be performed in reasonably short times even for values of the parameters close to the critical ones.

To this purpose, consider the "shaken dynamics" introduced in [3]. This dynamics can be seen as a dynamics on $\mathcal{X}_{\mathbb{H}}$ that, alternatively, updates the spins in $\Lambda^{1}$ and in $\Lambda^{2}$. In [3], Theorem 2.2, it has been shown that the equilibrium measure of the shaken dynamics, regarded as a dynamics on $\mathcal{X}_{\mathbb{H}}$, is the Gibbs measure $\pi_{2}$.

This parallel dynamics preserves the natural partial ordering between Ising configurations. Consequently, it allows to effectively exploiting massively parallel computing to draw unbiased samples from $\pi_{2}$ using perfect sampling techniques $[11,12]$. To draw a sample from $\pi$ it is, therefore, enough to draw a configuration from $\pi_{2}$ and look at the sub-configuration on the layer $\Lambda^{1}$. A more detailed numerical analysis of the shaken dynamics will be the topic of a forthcoming paper [2]. 


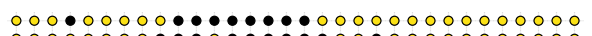

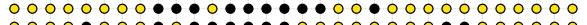

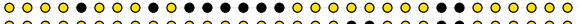
$0000000000000000000 \cdot 000: 000000$ $00000000-000000000000000: 000000$ $00000000000000000000000-000000$ $0-00000000000000000-00000-000000$

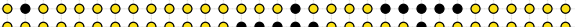

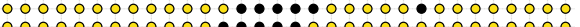
$000000000000 \cdot \because 00-000000000000$

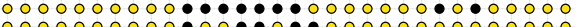

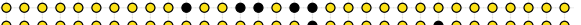

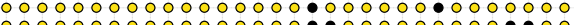

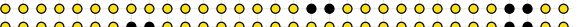
$0000000-00000000000000000-00$

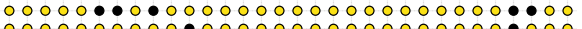

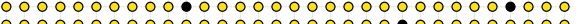

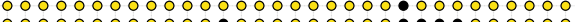

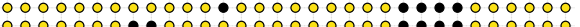

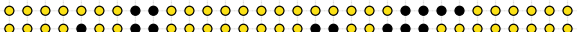

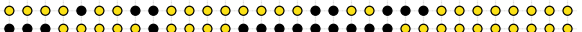

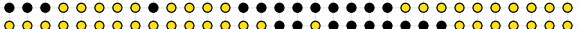
$000000000000000: 0: \because: \because: 0000000$ - $000000000000: \cdots: \because: \because: 000000$

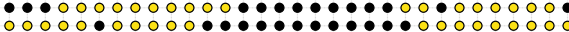

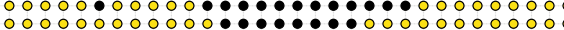

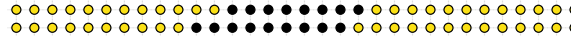

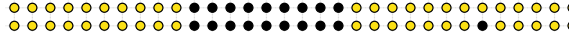

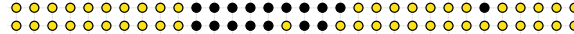

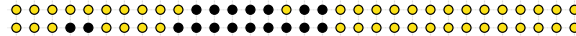
$00: 00000:::: 1: 00000000000000$

(a)

Fig. $5 J=0.44, q=3.0$

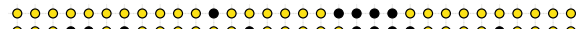

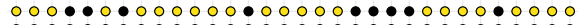
$0000: 00000000000$ : : : : :000000 $0-0-0-0-0-0-0-0-0-0-1-0-0-0-0-0$ $000000000000000: 1: 000000$

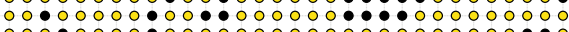

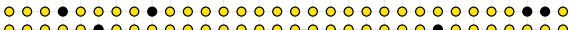

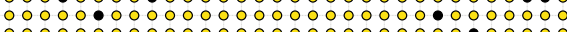
$000000000000000000000000-00000$

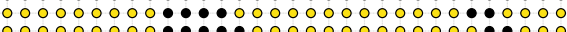
$0-0-0-0-0-0-:-0-0-0-0-0-0-0-0-0-0$ $00000000000-000000000000000000$

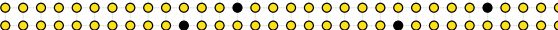

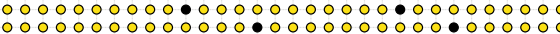

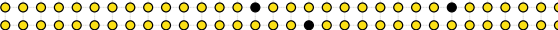

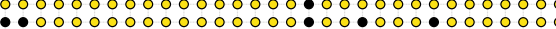
- $00-0-0-00-0-0-0: 0-0,0-0-0-0-0$ $0000000000000: 000000000$ $00000 \cdot 00000000:-1: 000000$

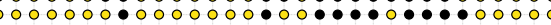
0 -0000000000000-0:-::-0.00 $00-0-0000000000-\cdots:-0:-0000$

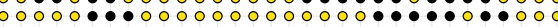
$0-0-0-0-0-0-0-0-0-0-0-0-0-0-0-0-0-0-0$ 00000000000000000 : :1:000

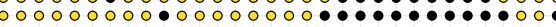

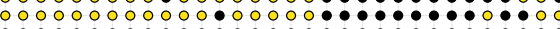

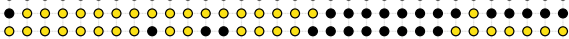

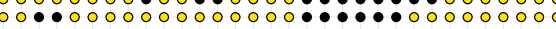

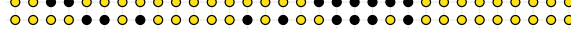

(a) $\because \because \because \because \because \because \because \because \because 0000000000000000$

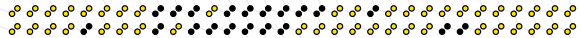

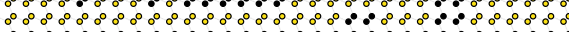

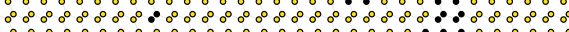

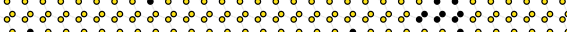

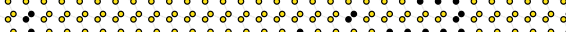

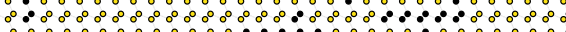

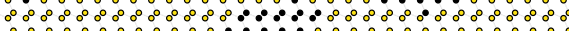

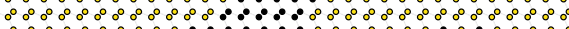

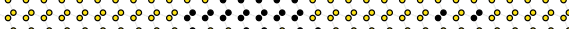

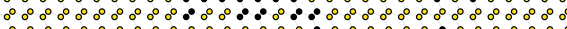
$\therefore \circ \therefore 000000000000000000000000$

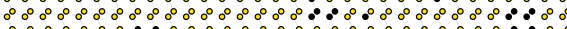

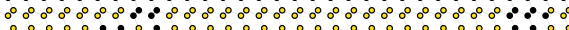

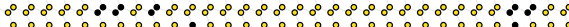

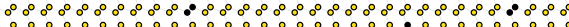

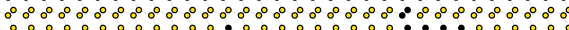

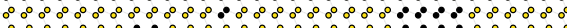
$\therefore \circ 0 \circ 000000000000000000000$ $\therefore \circ 0 \circ 0000000000000000000000$

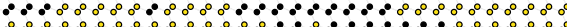

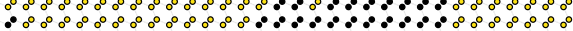

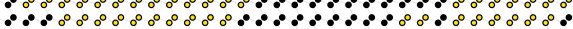

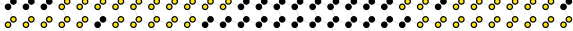

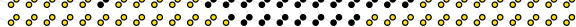

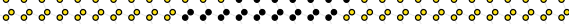

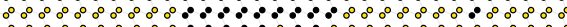

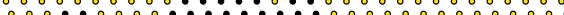

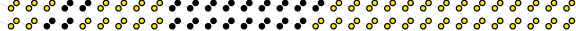

(b)

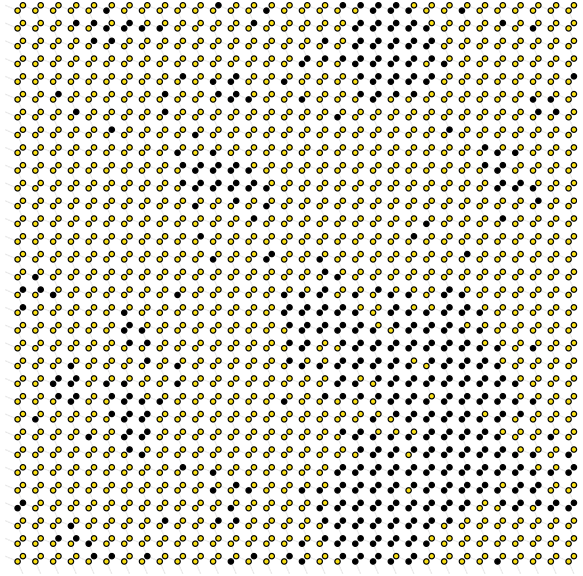

(b)

Fig. $6 J=0.6585, q=0.6585$

Figures 5, 6 and 7 show samples from the measure $\pi$ and the corresponding samples from the Gibbs measure $\pi_{2}$ on $\mathcal{X}_{\mathbb{H}}$ for several pairs of values of $J$ and $q$ near the critical curve.

Furthermore, numerical simulations show that the responsiveness of the "shaken dynamics" introduced in [3] to variations of the parameters is in very good accordance with the theoretical results of Theorem 2.2. For instance, looking at the average magnetization over a large number of iterations, it is possible to see that the parameter space is clearly split into two regions corresponding to the ordered and disordered phase. The numerically determined curve separating these two phases strongly agrees with the theoretical one (13) as shown in Fig. 8. 


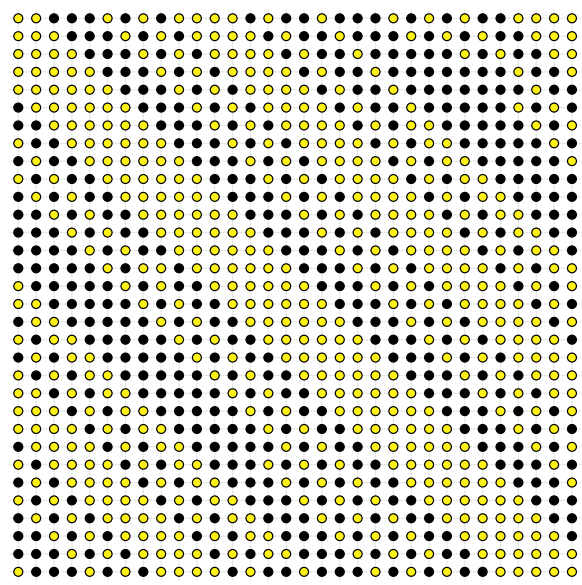

(a)

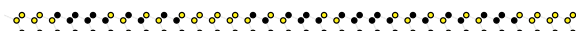

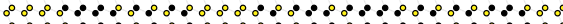

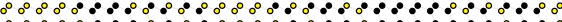
0000000000 $\therefore 00000000$ $\because 0 \%$

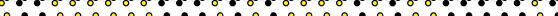
0

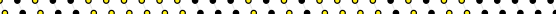

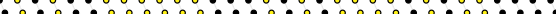

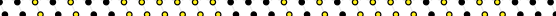

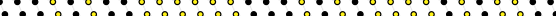

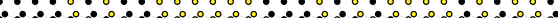

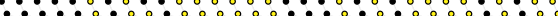
0

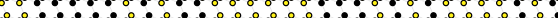
0 0

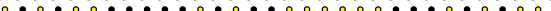

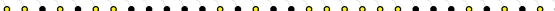
000

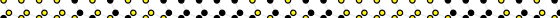
$\because 0000000000000000000$

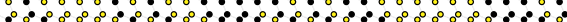
$\therefore 0000000000000000000$

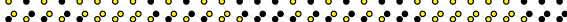

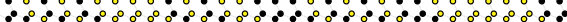

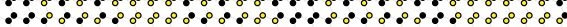

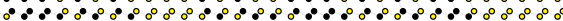

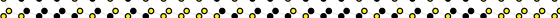

(b)

Fig. $7 J=2.0, q=0.03$

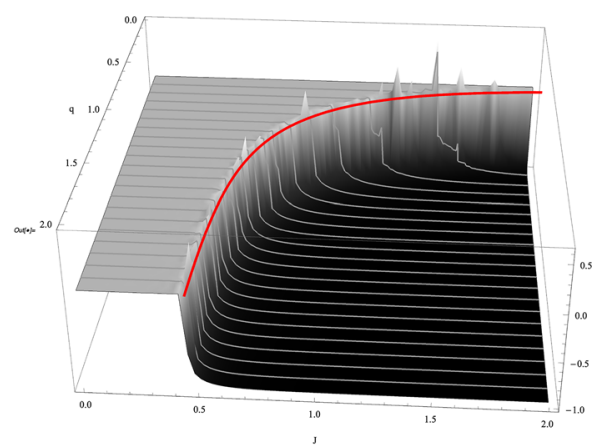

(a)

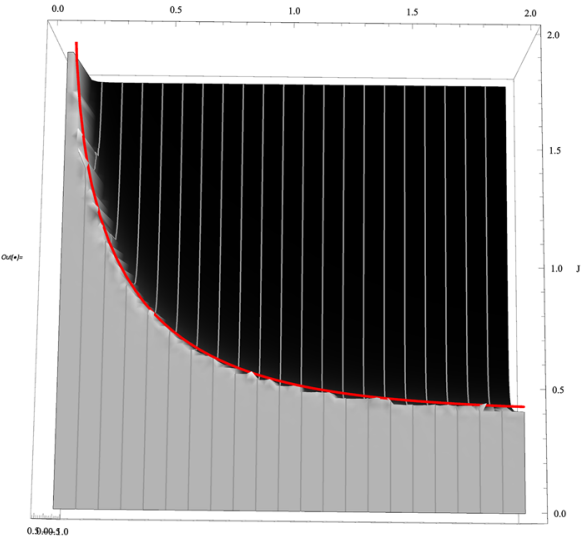

(b)

Fig. 8 The numerically determined critical curve

Acknowledgements We are grateful to Hugo Duminil-Copin for useful and interesting discussions. B.S. acknowledges the MIUR Excellence Department Project awarded to the Department of Mathematics, University of Rome Tor Vergata, CUP E83C18000100006. E.S. has been supported by the PRIN 20155PAWZB "Large Scale Random Structures". A.T. has been supported by Project FARE 2016 Grant R16TZYMEHN. B.S. and E.S. thank the support of the A*MIDEX Project (n. ANR-11-IDEX-0001-02) funded by the "Investissements d'Avenir" French Government program, managed by the French National Research Agency (ANR).

\section{References}

1. Aizenman, M., Chayes, J.T., Chayes, L., Newman, C.M.: Discontinuity of the magnetization in onedimensional $1 /|x-y|^{2}$ Ising and Potts models. J. Stat. Phys. 50, 1-40 (1988) 
2. Andrianaivo, L.N., D’Autilia, R., Troiani, A.: Parallel simulation of two-dimensional Ising models using Probabilistic Cellular Automata. (2019). arXiv:1908.07341

3. Apollonio, V., D’Autilia, R., Scoppola, B., Scoppola, E., Troiani, A.: Shaken dynamics for $2 d$ Ising models. arXiv: 1904.06257

4. Cimasoni, D., Duminil-Copin, H.: The critical temperature for the Ising model on planar doubly periodic graphs. Electron. J. Prob. 18(44), 1-18 (2013)

5. Dai Pra, P., Scoppola, B., Scoppola, E.: Sampling from a Gibbs measure with pair interaction by means of PCA. J. Stat. Phys. 149, $722-737$ (2012)

6. Dai Pra, P., Scoppola, B., Scoppola, E.: Fast mixing for the low-temperature 2D Ising model through irreversible parallel dynamics. J. Stat. Phys. 159, 1-20 (2015)

7. Edwards, R.G., Sokal, A.D.: Generalization of the Fortuin-Kasteleyn-Swendsen-Wang representation and Monte Carlo algorithm. Phys. Rev. D 38, 2009-2012 (1988)

8. Fortuin, C.M., Kasteleyn, P.W.: On the random cluster model. I. Introduction and relation to other models. Physica 57, 536-564 (1972)

9. Grimmett, G.R.: The Random-Cluster Model, volume 333 of Grundlehren der Mathematischen Wissenschaften [Fundamental Principles of Math Sciences]. Springer, Berlin (2006)

10. Procacci, A., Scoppola, B., Scoppola, E.: Probabilistic Cellular Automata for the low-temperature 2d Ising model. J. Stat. Phys. 165, 991-1005 (2016)

11. Propp, J., Wilson, D.: Exact sampling with coupled Markov chains and applications to statistical mechanics. Random Struct. Algorithms 9, 232-252 (1996)

12. Wilson, D.: How to couple from the past using read-once source of randomness. Random Struct. Algorithms 16, 85-113 (2000)

Publisher's Note Springer Nature remains neutral with regard to jurisdictional claims in published maps and institutional affiliations. 